\title{
Characterisation of cell adhesion in airway epithelial cell types using electric cell- substrate impedance sensing
}

\author{
I.H. Heijink*,\#, S.M. Brandenburg*, J.A. Noordhoek*, D.S. Postma", \\ D-J. Slebos ${ }^{\#}$ and A.J.M. van Oosterhout*
}

ABSTRACT: Research on epithelial cell lines and primary epithelium is required to dissect the mechanisms underlying the structural abnormalities in airway epithelium observed for respiratory diseases, including asthma and chronic obstructive pulmonary disease.

The novel electric cell-substrate impedance sensing technique was used to monitor cell adhesion/spreading, barrier function and wound healing. Primary bronchial epithelium was compared with airway epithelial cell lines 16HBE140-, BEAS-2B, NCI-H292 and A549.

BEAS-2B, A549 and primary cells form a confluent monolayer more rapidly than do 16HBE140cells. In contrast, 16HBE140- cells form stronger intercellular contacts, with a 10-fold higher resistance than BEAS-2B, A549 and $\mathrm{NCl}-\mathrm{H} 292$ cells and a five-fold increase over primary cells. Accordingly, expression of the adhesion molecules zona occludens-1 and E-cadherin was highest in 16HBE140- cells. These molecules were localised in intercellular junctions in both 16HBE140and primary cells. Finally, restoration of barrier function upon injury was impaired in BEAS-2B compared to 16HBE140- cells.

In conclusion, epithelial cell types display remarkable phenotypic differences and should, accordingly, be used to address specific research questions. 16HBE140- cells appear most suitable for studies on barrier formation, whereas resemblance in attachment of primary and BEAS-2B and A549 cells makes the latter more important for translational research on cell-matrix contact.

KEYWORDS: Adhesion molecules, airway epithelial injury, epithelial repair, respiratory disease, tight junctions

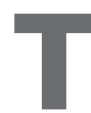
he airway epithelium plays a central role in the pathogenesis of various pulmonary diseases, including asthma, chronic obstructive pulmonary disease (COPD) and cystic fibrosis. In healthy subjects, the respiratory epithelium acts as a physical barrier to harmful inhaled substances, e.g. allergens, cigarette smoke and pathogens. Epithelial barrier function is maintained by intercellular contact formation in so-called adherens junctions (AJs) and tight junctions (TJs). AJs regulate adhesion through homotypic interactions of the adhesion molecule E-cadherin on adjacent cells, providing the architecture required for TJs [1]. TJs are localised more apically, comprise the interacting proteins occludin, claudins and zona occludens (ZO)-1 and restrict paracellular permeability. Under pathological conditions, disruption of epithelial integrity and/or inefficient epithelial repair may lead to increased access of environmental factors, e.g. allergens, to subepithelial layers. Indeed, the airway epithelium is often damaged in asthma, with increased permeability and loss of Ecadherin and ZO-1 expression [2-4]. Disruption of the epithelial barrier may also relate to structural abnormalities and epithelial remodelling, i.e. mucous hyperplasia and squamous cell metaplasia, as observed in COPD $[5,6]$.

Rapid restoration of the epithelial barrier upon injury is crucial, and may be distorted in patients suffering from respiratory diseases such as asthma and COPD. Complete regeneration of the airway epithelium requires epithelial migration, proliferation and reconstitution into a welldifferentiated epithelium that forms functional intercellular junctions. Better characterisation of cell adhesion during growth and repair processes is thus pivotal to the search for new therapeutic strategies. Moreover, it is of importance to use a cell line with high translational value for the in vivo situation. Since different cell lines vary in
AFFILIATIONS

*Laboratory of Allergology and Pulmonary Diseases, Dept of Pathology and Medical Biology, and \#Dept of Pulmonology, University Medical Center Groningen, University of Groningen, Groningen, the Netherlands.

CORRESPONDENCE

I.H. Heijink

Laboratory of Allergology and Pulmonary Diseases, Dept of Pathology and Medical Biology, University Medical Center Groningen University of Groningen

Hanzeplein 1

NL-9713 GZ

Groningen

The Netherlands

E-mail: h.i.heijink@int.umcg.nl

Received:

April 212009

Accepted after revision:

Sept 012009

First published online:

Sept 092009 
their capacity to form cell-cell contacts [7], the adhesive abilities of the bronchial epithelial cell lines 16HBE14o- and BEAS-2B, the mucoepidermoid carcinoma cell line NCI-H292, the alveolar type-II carcinoma cell line A549 and primary bronchial epithelial cells (PBECs) were compared. Epithelial resistance (Rep) was measured using electric cell-substrate impedance sensing (ECIS), a novel technique that permits accurate real-time monitoring of Rep, with the ability to distinguish between different processes that contribute to changes therein, i.e. cell attachment and formation of intercellular junctions [8]. In addition, the ECIS system was used to reproducibly and quantitatively study differences in epithelial repair and barrier recovery upon wounding by electroporation. Finally, whether differences between airway epithelial cell types relate to differences in the expression of cell-cell contact molecules was studied.

\section{METHODS}

\section{Cell culture}

The human bronchial epithelial cell line 16HBE14o- was kindly provided by D.C. Gruenert (University of California, San Francisco, CA, USA) and cultured in Eagle minimum essential medium (EMEM) $/ 10 \%$ fetal calf serum (FCS; Biowhittaker, Verviers, Belgium) on collagen-coated flasks as described previously [9]. Human bronchial BEAS-2B cells were cultured in RPMI medium $/ 10 \%$ FCS on collagen-coated flasks. The carcinoma cell lines NCI-H292 and A549 were cultured on uncoated flasks in RPMI medium $/ 10 \%$ FCS. All culture media were supplemented with $100 \mathrm{U} \cdot \mathrm{mL}^{-1}$ penicillin and $100 \mu \mathrm{g} \cdot \mathrm{mL}^{-1}$ streptomycin. PBECs from bronchial brushings in asthma, COPD and healthy control subjects were obtained and cultured as described previously [10-12]. Cells were cultured in hormonally supplemented bronchial epithelial growth medium (BEGM; Lonza, Walkersville, MD, USA), in flasks coated with collagen and fibronectin [13], and used for experiments at passage 2. Epithelial cells from healthy donors were used for both ECIS and immunodetection/staining $(n=3)$. Epithelial cells derived from COPD and asthma patients were used for either immunodetection/staining $(n=3)$ or ECIS $(n=1)$.

\section{ECIS}

The resistance of cells in culture was measured using ECIS, as described previously [8]. All tested cell lines were grown on collagen-coated arrays, whereas primary cells were grown on fibronectin-plus-collagen-coated arrays. Cell adhesion measurements were based on changes in resistance/capacitance to current flow applied to the electrode arrays at different frequencies (Applied Biophysics, Troy, NY, USA). A frequency scan was performed to test for the frequency at which the greatest difference in Rep was obtained between the cellcovered and cell-free electrodes. The baseline was established using culture medium $\left(400 \mu \mathrm{L} \cdot\right.$ well $\left.^{-1}\right)$ alone and compared with values obtained using electrodes covered with a monolayer of cells in $400 \mu \mathrm{L}$ medium. The optimal frequency for studying resistance appeared to be $400 \mathrm{~Hz}$. For the resistance/ capacitance measurements, cells were inoculated at $7.5 \times 10^{4}$ cells $\cdot$ well $^{-1}$, unless otherwise indicated, in $400 \mu \mathrm{L}$ in duplicate. Wounding was performed by electroporation using voltage pulses of $5 \mathrm{~V}$ and $40 \mathrm{kHz}$ for $30 \mathrm{~s}$.

\section{Air-liquid interface}

For air-liquid interface (ALI) cultures, cells were grown to

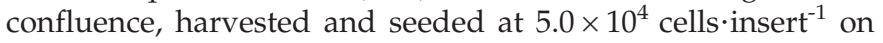
semi-permeable membranes (Transwell; Corning Incorporated, Cambridge, MA, USA; $0.4 \mu \mathrm{m}$ pore size; $12 \mathrm{~mm}$ diameter) coated with $30 \mathrm{~g} \cdot \mathrm{mL}^{-1}$ collagen and $10 \mu \mathrm{g} \cdot \mathrm{mL}^{-1}$ bovine serum albumin (BSA). Cells were cultured in a 1:1 mixture of Dulbecco's modified Eagle medium (Lonza) and BEGM supplemented with retinoic acid (Sigma, St Louis, MO, USA; $15 \mathrm{ng} \cdot \mathrm{mL}^{-1}$ ) and grown submerged until confluence (47 days), after which they were exposed to an ALI for another 2-4 weeks. Mucociliary differentiation was observed between days 14 and 26 after exposure to the ALI. Rep was measured using a voltohmmeter (EVOM; World Precision Instruments, Sarasota, FL, USA), which differs from ECIS in surface area $\left(1.12 \mathrm{~cm}^{2}\right.$ for the porous membrane insert versus $5 \times 10^{-4} \mathrm{~cm}^{2}$ for the electrodes in the ECIS system), applied current (20 versus $1 \mu \mathrm{A}$ in the ECIS system) and the inability to measure at different frequencies and thereby distinguish between cell-cell and cell-matrix contacts.

\section{Small interfering RNA downregulation of E-cadherin}

E-cadherin gene expression was downregulated in the 16HBE14o- cells by use of small interfering RNA (siRNA), as described previously [9]. In brief, cells were seeded at

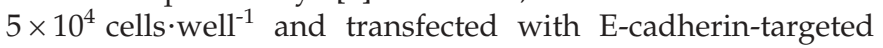
siRNA/nontargeting control oligonucleotides (Eurogentec, Liège, Belgium) at a final concentration of $20 \mu \mathrm{M}$ using Oligofectamine (Invitrogen Corporation, Carlsbad, CA, USA).

\section{Immunodetection by Western blotting}

Total cell lysates were obtained by resuspension of the cells in Laemmli buffer, and immunodetection was performed as described previously [9], using anti-E-cadherin (Santa Cruz Biotechnology, Santa Cruz, CA, USA) and anti-ZO-1 (Zymed, San Francisco, CA, USA).

\section{Immunofluorescence staining}

Cells grown on Lab-Teks (Nunc, Rochester, NY, USA) were washed with $\mathrm{PBS} / \mathrm{CaCl}_{2}$, fixed in ice-cold acetone $(90 \%)$ for $30 \mathrm{~min}$, blocked in $\mathrm{PBS} / 5 \% \mathrm{BSA}$ for $60 \mathrm{~min}$, incubated for 60 min with primary antibodies (1:200) and then subsequently incubated for $60 \mathrm{~min}$ with fluorescein-isothiocyanate-labelled anti-rabbit (1:200, Dako Diagnostics, Mississauga, ON, Canada) or rhodamine-labelled anti-mouse immunoglobulin G conjugates (Jackson Immunoresearch Laboratories, West Grove, PA, USA; 1:400) or rhodamine-labelled phalloidin (Cytoskeleton, Inc., Denver, CO, USA; 1:200) in the presence of 4',6-diamidino-2-phenylindole (1:500) and analysed as described previously [14].

\section{RESULTS}

\section{Characteristics of 16HBE140- attachment and cell-cell contact formation}

Human bronchial epithelial 16HBE140- cells were first tested by monitoring the capacitance of the monolayer at a high frequency $(40 \mathrm{kHz})$ and the resistance at a low frequency (400 Hz). The Rep and the current flow in the adhesion area of the cells are dependent on the frequency of the applied current. WEGENER et al. [8] have shown that, when monitoring growth and attachment, resistance is most sensitively measured at a 
low frequency (low-frequency resistance). At low frequencies $(200-5,000 \mathrm{~Hz})$, the resistance increased during the initial attachment and spreading phase, going through a period of slow increase and then rising to the final levels, which are thought to be due to the establishment of TJs. The capacitance measurements, conversely, appeared relatively insensitive to changes at low frequencies, becoming more sensitive to changes due to cell attachment/spreading when moving to higher frequencies. In contrast to the resistance, the capacitance measurements were barely affected by the formation of intercellular junctions. These data suggest that changes in high-frequency capacitance are most probably caused by cell attachment, spreading and growth, and that the low-frequency changes, when not paralleled by changes in high-frequency capacitance, are most probably the consequence of intercellular junction formation.

Cells inoculated at a density of $7.5 \times 10^{4}-10.0 \times 10^{4}$ cells $\cdot$ well ${ }^{-1}$ reached confluence after $30-50 \mathrm{~h}$, as established by an initial decrease followed by stabilisation of the high-frequency capacitance (fig. 1b), which is inversely related to resistance. During the initial spreading/attachment of the cells within the first $40 \mathrm{~h}$, only a minimal increment could be observed in the low-frequency resistance (fig. 1a), and the marked increase upon stabilisation of the capacitance between $40-60$ and $80 \mathrm{~h}$ most probably reflected the establishment of intercellular contacts. In order to confirm that the increase in low-frequency resistance during this period was a reflection of the formation of intercellular junctions, the localisation of the junctional proteins ZO-1 and E-cadherin upon inoculation were studied. As expected, a continuous circumferential localisation of both ZO-1 and E-cadherin was observed after $72 \mathrm{~h}$, whereas junctional expression of these proteins was still fragmented after $24 \mathrm{~h}$ (fig. 1c-f).

At a density of $2.5 \times 10^{4}-5.0 \times 10^{4}$ cells $\cdot$ well $^{-1}$, confluence was reached at $60-80 \mathrm{~h}$, as confirmed by visual microscopic analysis (data not shown); nevertheless, the cells were not able to form tight intercellular junctions efficiently within the time-frame of the present experiment, as demonstrated by the low-frequency resistance (fig. 1a). Therefore, inoculation of $7.5 \times 10^{4}$ cells $^{\prime}$ well $^{-1}$ was used for all further experiments described in the present manuscript.

\section{E-cadherin-mediated cell-cell contact formation and low- frequency resistance}

In order to confirm the involvement of intercellular junctions in the increase in low-frequency resistance of 16HBE14o- cells, the changes upon siRNA downregulation of E-cadherin were monitored. The capacitance at a high frequency $(40 \mathrm{kHz})$, which should be relatively insensitive to changes in cell-cell contact, was also measured. E-cadherin expression was already effectively downregulated at day 1 following transfection and remained downregulated during the course of the experiment ( $\leqslant 72 \mathrm{~h}$ ) (fig. 2c). Low-frequency resistance increased from 24 to $60 \mathrm{~h}$ in the control cells (fig. 2a). Cells transfected with Ecadherin-targeted siRNA showed a far less pronounced increase (fig. 2a). High-frequency capacitance measurements, conversely, were not affected by the downregulation of E-cadherin (fig. 2b), indicating that loss of E-cadherin-mediated junctions specifically affects low-frequency resistance measurements. TJ sealing has been reported as a major determinant in electrical resistance [15], whereas prior formation of AJs may be required for proper assembly of TJs [16]. Therefore, expression of the TJ protein ZO-1 was analysed and reduced levels observed upon siRNA downregulation of E-cadherin (fig. 2d).

In order to find further support for the sensitivity of lowfrequency resistance to the formation of intercellular junctions, the 16HBE14o-monolayer was depleted of $\mathrm{Ca}^{2+}$, since epithelial junctions are completely $\mathrm{Ca}^{2+}$-dependent. $\mathrm{Ca}^{2+}$ deprival resulted in a dramatic decrease in low-frequency resistance towards the levels of uncovered electrodes (fig. 2e). In accordance, immunofluorescence staining revealed a complete loss of junctional expression of E-cadherin (fig. $2 \mathrm{f}$ and $\mathrm{g}$ ).

\section{Comparison of 16HBE140-, BEAS-2B and H292 cells and PBECs}

It was of interest to study the specific adhesive characteristics of various human airway epithelial cells, and marked differences were observed. BEAS-2B cells were relatively inefficient regarding the formation of intercellular junctions compared to $16 \mathrm{HBE} 14 \mathrm{O}-$ cells, as reflected in a 10-fold lower resistance (fig. 3a). In contrast, BEAS-2B cells established a confluent monolayer more rapidly, with a strong decline in capacitance within $3 \mathrm{~h}$ after inoculation (fig. 3b). After stabilisation of the capacitance, the low-frequency resistance increased only marginally to a maximum of an $\sim 2.5$-fold increase, in contrast to the strong increase in barrier tightness of the $16 \mathrm{HBE} 14 \mathrm{o}-$ cells. A549 cells showed similar characteristics to BEAS-2B cells, with rapid formation of a confluent layer. After this, low-frequency resistance did not increase further, suggesting that the initial increase occurred merely due to coverage of the electrodes, without formation of TJs (fig. 3c). NCI-H292 cells were also inefficient in establishing intercellular junctions (fig. $3 e$ and $\mathrm{f}$ ), with a slow and weak increase in low-frequency resistance over time that followed the kinetics of high-frequency capacitance.

Of all the tested cells types, PBECs showed the most rapid initial decrease in capacitance, reaching a minimum within $2 \mathrm{~h}$ (fig. 3h). Remarkably, this was followed by a temporary increase before values started to decline again (fig. 3h). These dynamics appear to be a specific hallmark of PBECs, and might be caused by alterations in cell shape, with initial attachment/ spreading followed by adoption of a less-flattened cell shape, e.g. to prepare for proliferation. During the first $48 \mathrm{~h}$, the primary cells showed only a modest increase in low-frequency resistance, with a similar fluctuation as observed for the highfrequency capacitance. The resistance markedly increased at 55-60 h (after cells were hormone/growth factor-deprived) (fig. 3g), resulting in a 4-7-fold increase over initial values (versus a 25-fold increase in 16HBE14o- cells). Finally, the Rep of PBECs grown at the ALI was measured, since PBECs can differentiate into a polarised mucociliary epithelium under these conditions [17], forming a tight epithelial barrier. It is not possible to measure the resistance of cells grown on trans-well membranes using ECIS. Therefore, a conventional voltohmmeter was used. Rep increased markedly in PBECs grown under ALI conditions, from $151-190 \Omega \cdot \mathrm{cm}^{2}$ at confluence under submerged conditions to up to $560-784 \Omega \cdot \mathrm{cm}^{2}$ upon mucociliary differentiation. 16HBE14o- cells, conversely, established Rep as high as $672-1,120 \Omega \cdot \mathrm{cm}^{2}$ (versus $60-100 \Omega \cdot \mathrm{cm}^{2}$ measured using the ECIS electrodes) when grown under 

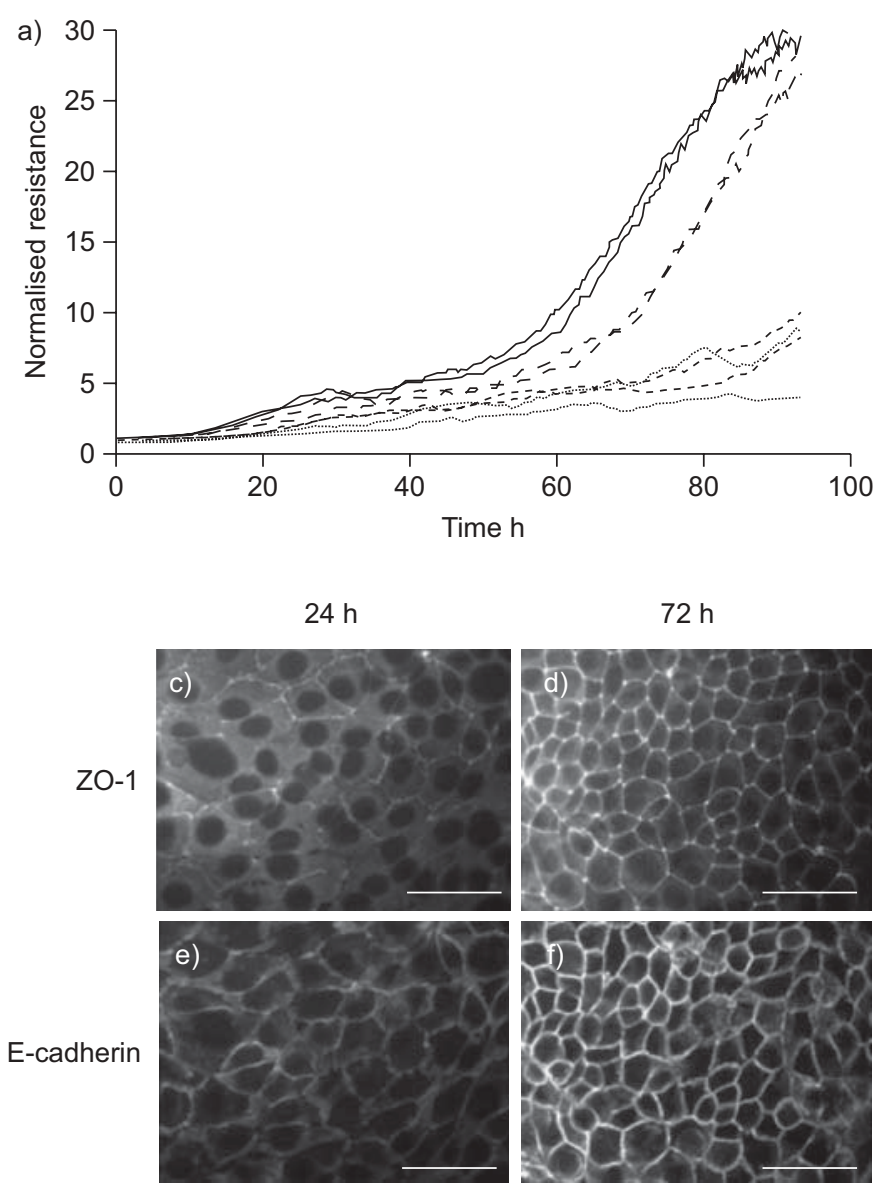

submerged conditions (EMEM/10\%FCS), but did not further increase their barrier tightness nor differentiate into mucociliary cells under ALI conditions (data not shown).

In conclusion, whereas PBECs adhere faster than 16HBE14ocells and resemble A549 and BEAS-2B cells more closely in this respect, the barrier tightness of PBECs is more comparable to that of 16 HBE14o- cells, but their barrier only becomes as tight as in 16HBE140- cells when PBECs are grown under ALI conditions.

\section{Epithelial junction formation and expression and localisation of cell adhesion molecules}

It was then tested whether or not differences in the barrier function of the individual monolayers were paralleled by expression of junctional proteins. In agreement with their barrier tightness, the ability of 16HBE14o- cells to form intercellular junctions was confirmed by the highest expression of ZO-1 (fig. 4a), whereas reduced levels of ZO-1 were observed in A549 and NCI-H292 cells and PBECs (healthy donor), and the lowest levels were expressed by BEAS-2B cells (fig. 4a). E-cadherin was highly expressed in 16HBE14o-, NCIH292 and primary cells, despite the lower capacity of NCIH292 to form intercellular junctions. BEAS-2B and A549 cells expressed low levels of E-cadherin (fig. 4a).

In order to validate whether or not the ECIS system can be a useful tool in the comparison of the adhesive characteristics of epithelial cell lines, epithelial cells derived from different

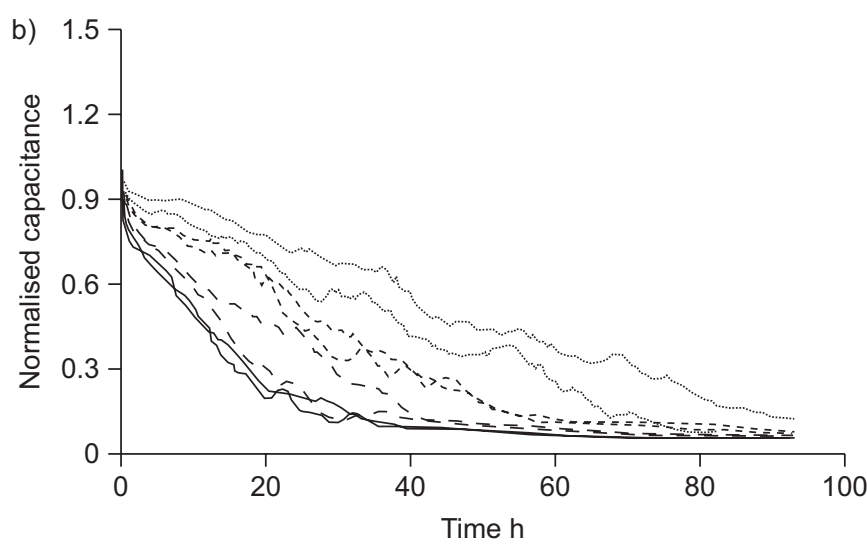

FIGURE 1. Attachment, growth and cell-cell contact formation characteristics of 16HBE140- cells. 16HBE140- cells were seeded in duplicate into electric cellsubstrate impedance sensing arrays at various concentrations (----

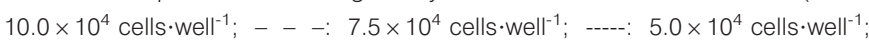

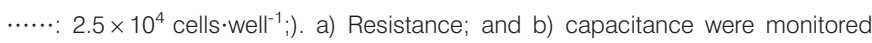
immediately after transfection, over a time course of $96 \mathrm{~h}$, at $400 \mathrm{~Hz}$ and $40 \mathrm{kHz}$, respectively. In order to correct for well-to-well variance, resistances and capacitances were normalised to the starting point at the uncovered electrode. Initial well-to-well variance was nonsignificantly different when cells were inoculated at different concentrations. The resistances of the uncovered electrodes typically ranged 500-700 $\Omega$ and capacitances ranged 60-80 nF. The results shown (in duplicate) are representative of three independent experiments. c-f) $7.5 \times 10^{4}$ cells well $^{-1}$ were grown on Lab-Teks for: c, e) 24; and d, f) 72 h; and c, d) zona occludens (ZO)-1; and e, f) E-cadherin and were detected by immunofluorescence staining. Scale bars $=50 \mu \mathrm{m}$.

individuals were studied. Similar levels of E-cadherin were observed in PBECs from asthma, COPD and healthy subjects. In contrast, the expression of ZO-1 was markedly reduced in asthma epithelium (fig. 4a; representative of three independent experiments). Accordingly, it was observed that low-frequency resistance was lowest in epithelial cells derived from an asthma patient compared to healthy and COPD cultures (fig. $4 \mathrm{~b}$; isolated observation). Although no conclusions can be drawn regarding the epithelial barrier function of asthmatics from this experiment, the present data indicate that ECIS is a promising tool for the quantification of differences in epithelial barrier tightness between different patient groups.

Since NCI-H292 cells expressed relatively high levels of ZO-1, despite the low barrier function, the localisation of ZO-1 and Ecadherin was also studied. Continuous circumferential localisation of ZO-1 and E-cadherin in intercellular junctions is characteristic of cells that form occluding junctions. As expected, this was observed for $16 \mathrm{HBE} 14 \mathrm{o}-$ cells (fig. $5 \mathrm{a}$ and b). In contrast, expression of ZO-1 and E-cadherin was fragmented and discontinuous in NCI-H292 cells, offering an explanation for the impaired ability to form a tight barrier (fig. $5 \mathrm{~d}$ and e). In BEAS-2B cells, E-cadherin was hardly detectable by immunostaining, although faint intercellular expression of ZO-1 could be observed (fig. $5 \mathrm{~g}$ and $\mathrm{h}$ ). This may account for the increased barrier tightness of BEAS-2B versus NCI-H292 monolayers. The localisation of E-cadherin and ZO-1 in A549 cells resembled the expression pattern of NCIH292 cells (fig. 5j and k). In PBECs, intercellular junctions were 

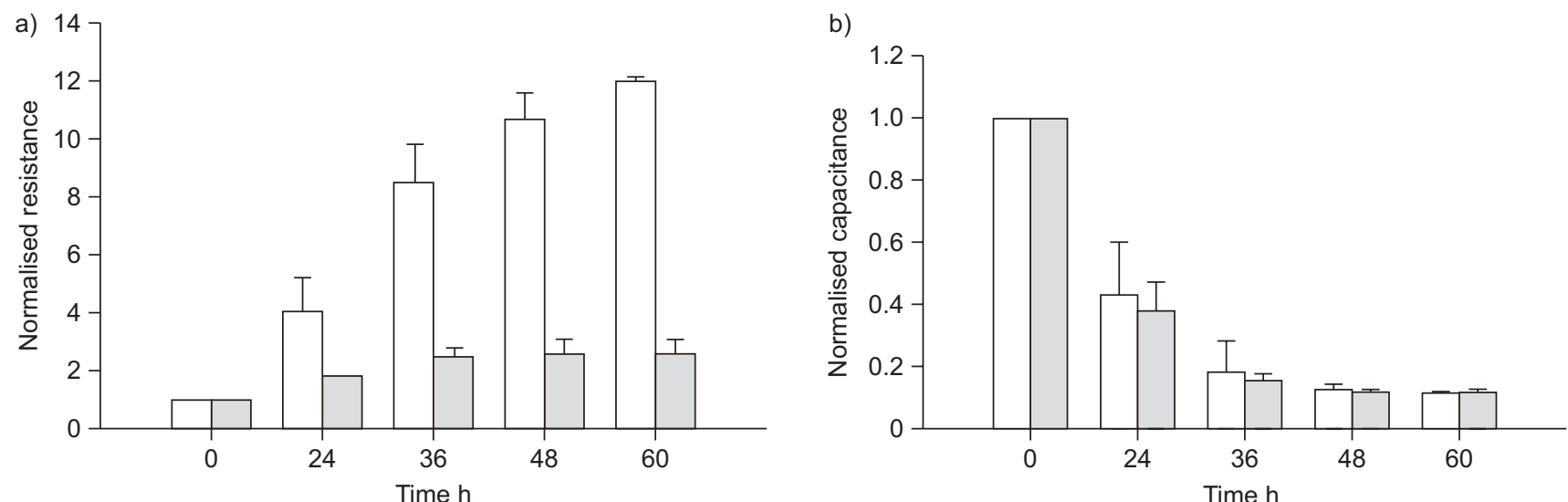

c)

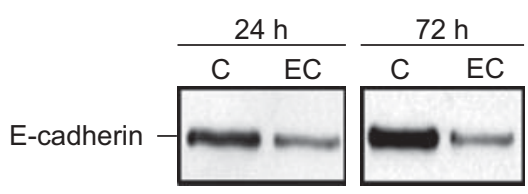

Basal
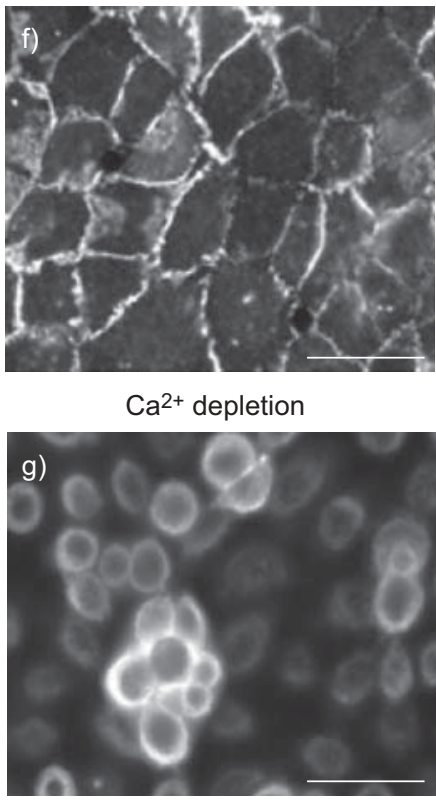

d)

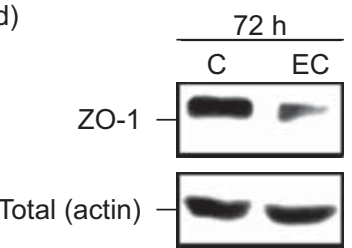

e)

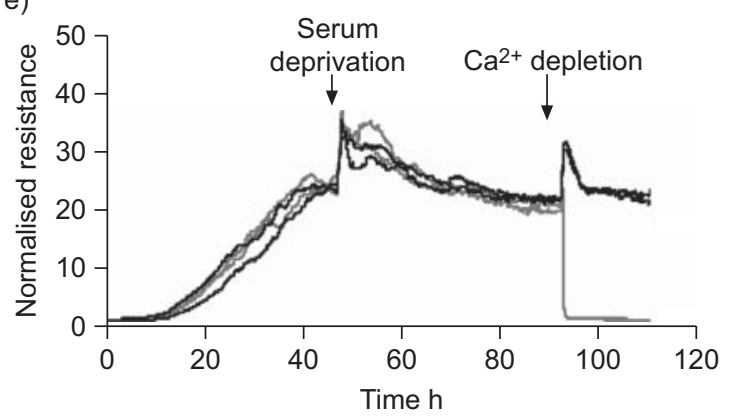

FIGURE 2. Reduced low-frequency resistance upon disruption of intercellular contacts. 16HBE140- cells were seeded at a density of $2.5 \times 10^{4}-10.0 \times 10^{4}$ cells $\cdot$ well $^{-1}$ into collagen-coated electric cell-substrate impedance sensing (ECIS) arrays and transfected with either the nontargeting small interfering RNA (siRNA) control $(\square ; C)$ or specific E-cadherin siRNA oligonucleotides ( $\square$; EC). On the following day, 16HBE14o- cells were seeded in triplicate into ECIS arrays at different concentrations or 24-well plates. a) Resistance; and b) capacitance were measured in triplicate 0-60 h after transfection by ECIS, at $400 \mathrm{~Hz}$ and $40 \mathrm{kHz}$, respectively. Data are presented as mean \pm SEM. c) $16 \mathrm{HBE} 14 \mathrm{O}$ - cells were seeded at a density of $2.5 \times 10^{4}$ $10.0 \times 10^{4}$ cells $\cdot$ well ${ }^{-1}$ in triplicate into collagen-coated 24-well plates and transfected with either $\mathrm{C}$ or EC. Total cell lysates were prepared after 24 and $72 \mathrm{~h}$, and E-cadherin was detected by Western blotting. d) zona occludens (ZO)-1 was detected by Western blotting in total cell lysates of cells transfected with either $\mathrm{C}$ or EC after $72 \mathrm{~h}$. e) Cells were seeded in duplicate (both shown) into ECIS arrays. After 48 h, when cells had reached confluence, the medium was replaced with serum-free medium. After $48 \mathrm{~h}, \mathrm{Ca}^{2+}$ was depleted (grey trace; by means of medium replacement) and resistance at $400 \mathrm{~Hz}$ measured (black trace: control). f, g) Cells were seeded in duplicate into ECIS arrays or Lab-Teks. After overnight serum deprivation, $\mathrm{Ca}^{2+}$ was (g) or was not (f) depleted for $10 \mathrm{~min}$. E-cadherin was detected by immunofluorescence staining. Scale bars $=50 \mu \mathrm{m}$.

present, as judged by the well-defined continuous expression of both ZO-1 and E-cadherin, although membrane expression of Ecadherin was less intense than in the 16HBE14o- cells, and ZO-1 did not appear to separate each adjacent cell (fig. $5 \mathrm{~m}$ and $\mathrm{n}$ ).

Dynamics in cytoskeletal actin filaments are thought to be the driving force of cell extension, flattening and attachment on the extracellular matrix [18]. Marked differences in cytoskeletal organisation of filamentous actin (F-actin) were observed between BEAS-2B, A549 and primary cells versus 16HBE14oand NCI-H292 cells. F-actin was assembled in a stress fibre-like pattern in PBECs and A549 and BEAS-2B cells, whereas it was mainly localised parallel to the membrane in 16HBE14o- and NCI-H292 cells (fig. 4c, f, i, 1 and o). These differences may be linked to the differential kinetics of cell spreading and attachment, as measured by high-frequency capacitance.

In conclusion, the extent and contiguity of E-cadherin, F-actin and, particularly, ZO-1 expression at intercellular junctions appears to correlate with the adhesive characteristics of the cells. Table 1 presents an overview of the adhesive characteristics of the cell lines. 

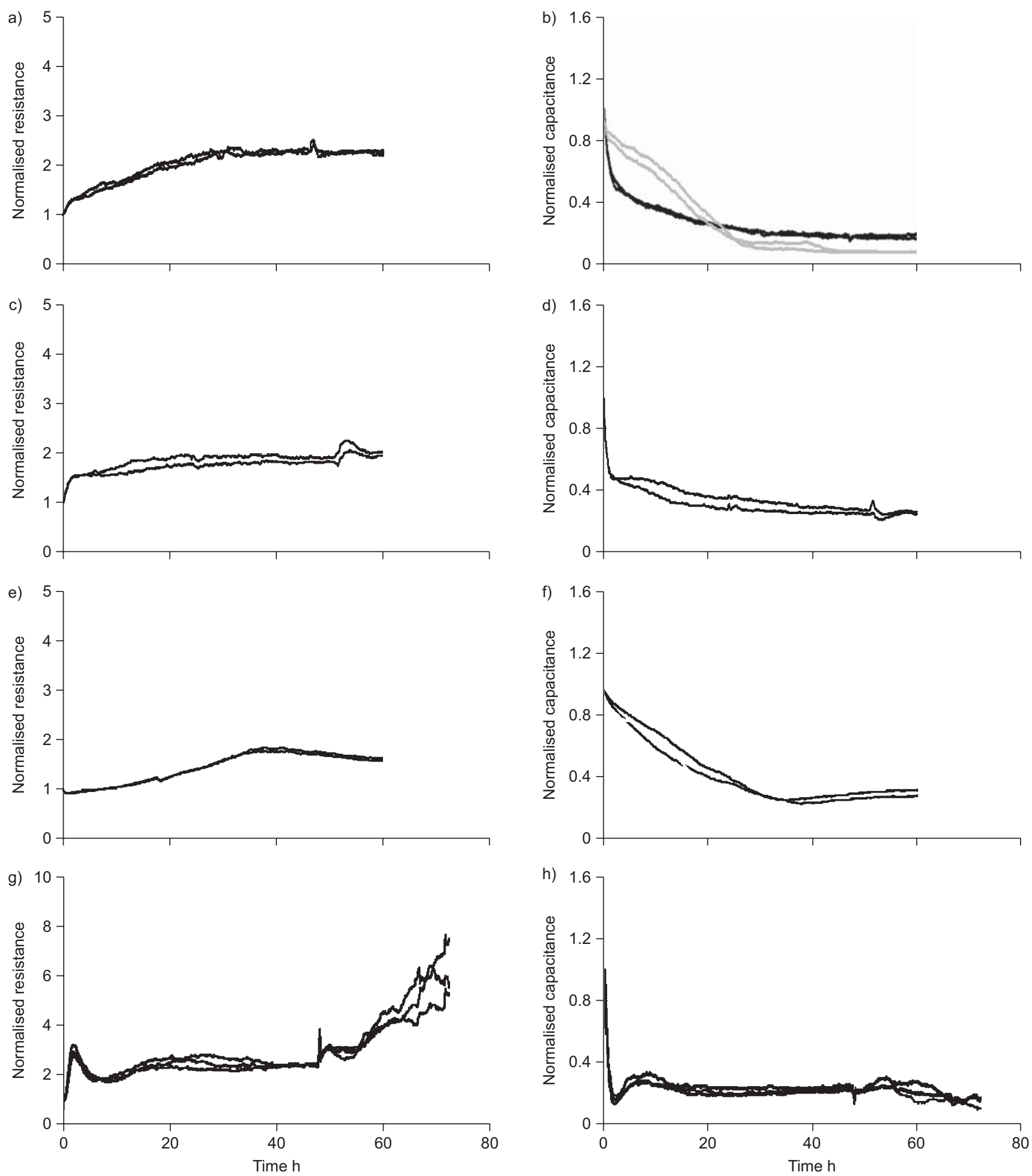

FIGURE 3. Attachment, growth and cell-cell contact formation characteristics of: a, b) BEAS-2B cells (grey trace:16HBE140- cells); c, d) A549 cells; e, f) NCI-H292 cells and $\mathrm{g}, \mathrm{h}$ ) primary bronchial epithelial cells (PBECs) derived from a healthy donor. Cells were seeded in duplicate (both shown) on to collagen-coated electric cell-substrate impedance sensing (ECIS) arrays. Resistance and capacitance were measured using ECIS immediately after seeding over a time course of $72 \mathrm{~h}$ at $400 \mathrm{~Hz}$ and $40 \mathrm{kHz}$, respectively. No differences were observed in the starting values of the different cell types upon inoculation (ranging 500-700 $\Omega$ and 60-80 nF, respectively); resistances and capacitances were normalised to the starting point at the uncovered electrode in order to correct for the well-to-well variance. For PBECs, the medium was replaced by hormone/growth factor-deprived medium (bronchial epithelial cell basal medium) after $48 \mathrm{~h}$. The results shown are representative of three independent experiments. 

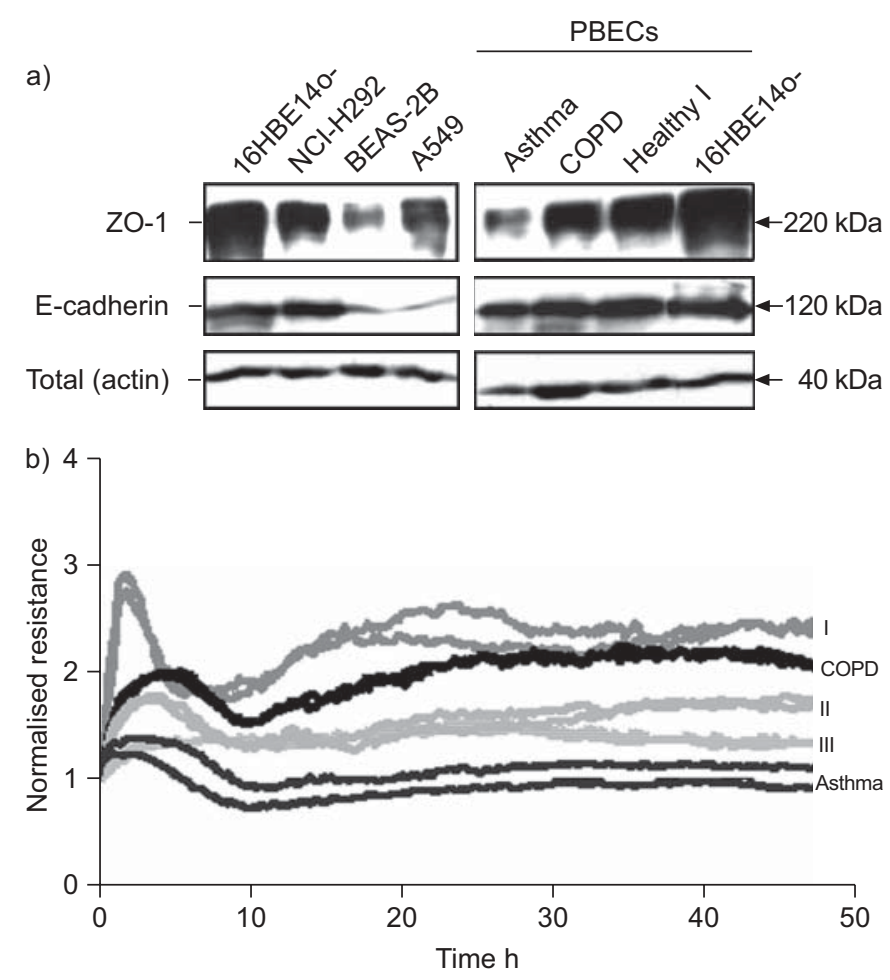

FIGURE 4. The ability to form epithelial junctions is related to the expression and localisation of cell adhesion molecules. a) 16HBE140-, NCI-H292, BEAS-2B and A549 cells or primary bronchial epithelial cells (PBECs) from asthma, chronic obstructive pulmonary disease (COPD) and healthy donors ( $\mathrm{n}=3 ; \mathrm{I}$, II and III) were seeded in duplicate in 24-well plates and cultured to confluence (48-72 h). Cells were serum/growth factor-deprived overnight and total cell lysates were prepared. Zona occludens (ZO)-1 and E-cadherin were detected by Western blotting. $\beta$-Actin was used as a control to ensure equal loading. b) PBECs from an asthma patient, a COPD patient and three healthy donors (I, II and III) were seeded in duplicate (both shown) into electric cell-substrate impedance sensing (ECIS) arrays. Normalised resistance was measured immediately after seeding over a time course of $60 \mathrm{~h}$ at $400 \mathrm{~Hz}$ using ECIS. Representative results are shown.

\section{Migration and recovery of epithelial barrier upon wounding}

In order to characterise the cell migration and regeneration response in BEAS-2B and 16HBE140- cells (which differed strongly in their cell-adhesive characteristics), electric-fieldinduced cell death was applied and the subsequent morphological wound healing response monitored. Using the ECIS system, the extent of the highly reproducible wound is restricted to the small $250-\mu \mathrm{m}$-diameter electrode, resulting in almost complete detachment of the cells from the electrode [19], as confirmed by microscopy following washing away of the dead cells (fig. 6e). This was accompanied by a dramatic drop in Rep and a corresponding increase in capacitance (fig. 6a and c). Within $2 \mathrm{~h}$, cells started to spread and migrate over the electrode again (fig. 6f), resulting in a decrease in capacitance (fig. 6c) and an increase in resistance (fig. 6a). Complete coverage of the electrode by the 16HBE14o- cells was reached within $2 \mathrm{~h}$, as measured by high-frequency capacitance. From the time that a confluent monolayer was reestablished, low-frequency resistance continued to increase slowly towards its original values, indicating reconstitution of intercellular junctions (fig. 6a). In contrast, BEAS-2B cells showed no further increase in low-frequency resistance once the capacitance had stabilised, which took $\sim 2 \mathrm{~h}$ longer than in 16 HBE14o- cells (fig. $6 \mathrm{~b}$ and d). This indicates that the increase in epithelial resistance occurs merely due to repopulation of the electrode, and that BEAS-2B cells are unable to restore their TJs upon this type of injury. Thus differences in the celladhesive characteristics of BEAS-2B and 16HBE140- are reflected in their wound healing response.

\section{DISCUSSION}

The present study showed that the ECIS system is a versatile tool for monitoring various aspects of cell adhesion in airway epithelial cells during growth and repair. Measurement of Rep is a useful substitute for permeability assays, with possible difficulties due to diffusion of the solute. Furthermore, a major advantage of ECIS is that noninvasive real-time measurements can be performed at different frequencies. It is demonstrated here that the high-frequency capacitance changes predominantly during initial cell spreading and attachment, but is hardly sensitive to alterations in intercellular junctions. The latter is most sensitively measured by resistance at a low frequency, enabling the distinction between intercellular and cell-matrix contacts.

Large differences in adhesive characteristics were shown between different airway epithelial cells, which can be related to the expression and/or localisation of ZO-1 and E-cadherin (table 1). Although A549, BEAS-2B and primary cells showed faster spreading/attachment, the greatest barrier tightness was constituted by 16 HBE14o- cells once confluence was reached. PBECs displayed moderate formation of $\mathrm{TJ}$ s and AJs, as reflected by the junctional expression of ZO-1 and E-cadherin. The present findings further suggest that $\mathrm{ZO}-1$ is a more important determinant in the formation of a tight epithelial barrier than E-cadherin. The isolated observation on the reduced formation of an epithelial barrier in asthma versus healthy epithelium is in agreement with the reduced expression of ZO-1, as also observed by others [2,3]. We will further study the barrier function of asthma patients in future studies.

The more rapid attachment of BEAS-2B, A549 and primary cells versus $16 \mathrm{HBE} 14 \mathrm{o}-$ and NCI-H292 cells may result from differences in the dynamics of actin filament organisation, which is thought to contribute to cell spreading/attachment responses [18]. Indeed, whereas F-actin showed a cortical localisation in 16HBE140- and NCI-H292 cells, stress fibres were observed in PBECs and BEAS-2B cells. In addition to its function in attachment, the F-actin cytoskeleton is thought to regulate cell motility [20]. The migratory response to injury appeared slower in BEAS-2B than in 16HBE14o- cells, suggesting that the observed F-actin arrangement in BEAS-2B cells favours cells attachment rather than cell motility.

Using ECIS for wounding, cells need only migration, but not proliferation, to repopulate the electrode, since the electrodes are relatively small. This allows not only for more relevant but also more precise study of the migration response compared to the scratch-wound method, where both proliferation and migration occur during the $24 \mathrm{~h}$ required to close the relatively large wound. In vivo, interaction with and remodelling of the extracellular matrix play a central role in epithelial cell migration, proliferation and restoration of the barrier during 
E-cadherin

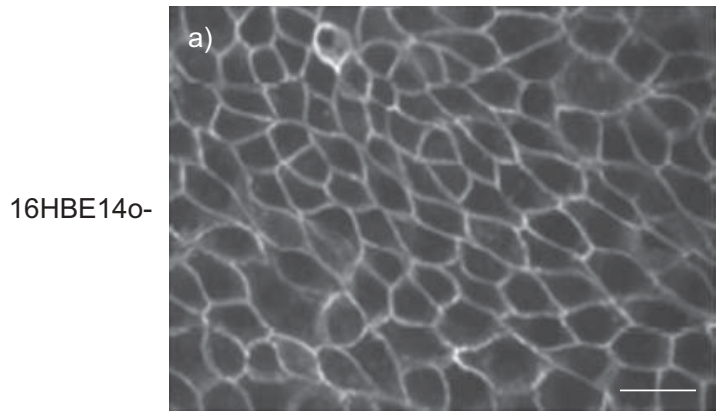

$\mathrm{NCl}-\mathrm{H} 292$

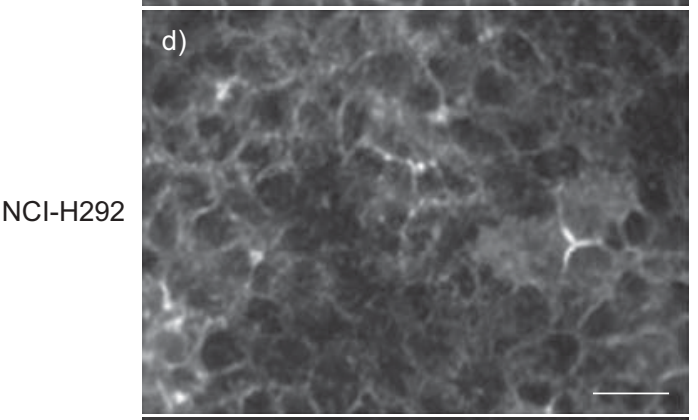

g)

BEAS-2B

A549

PBECs
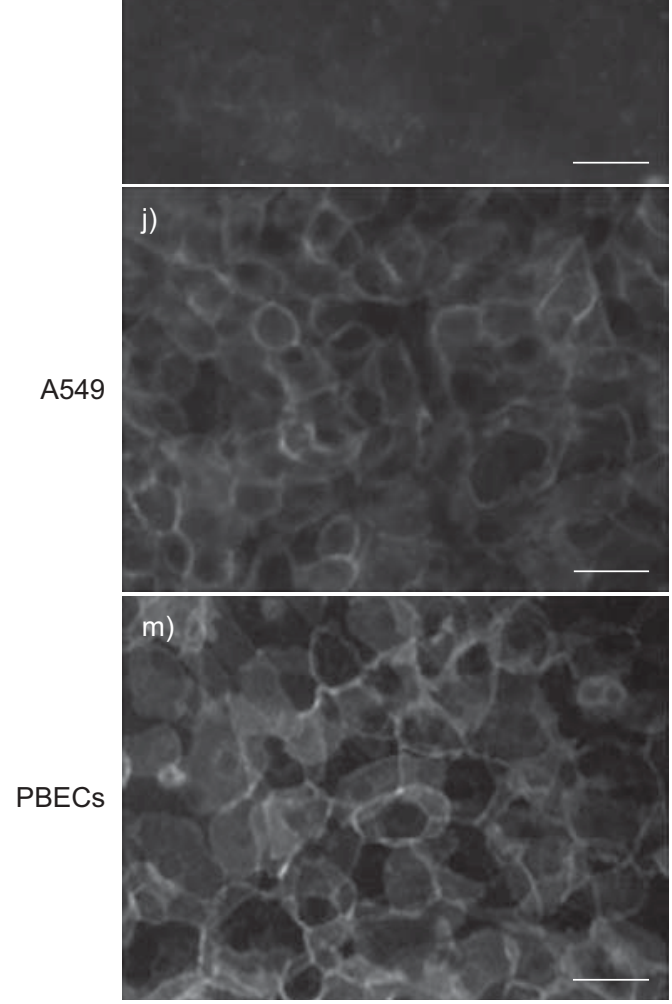

ZO-1
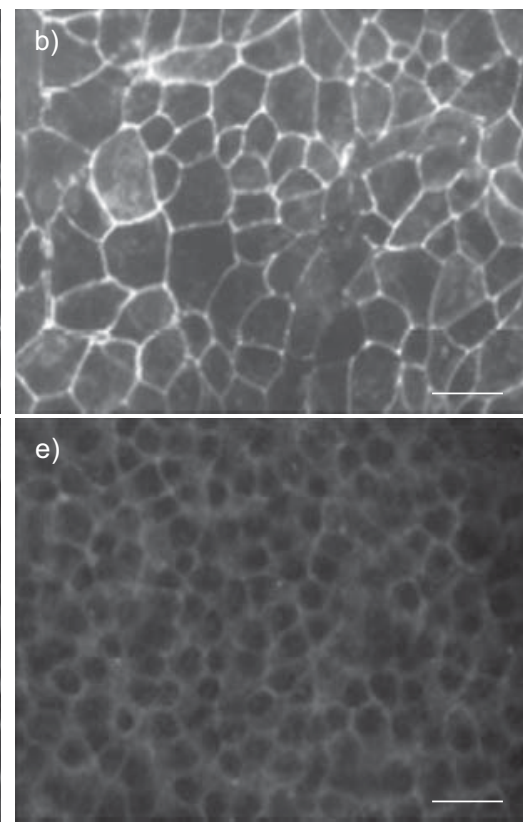

h)

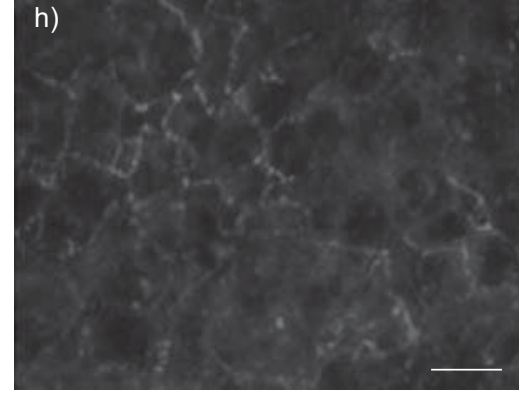

k)
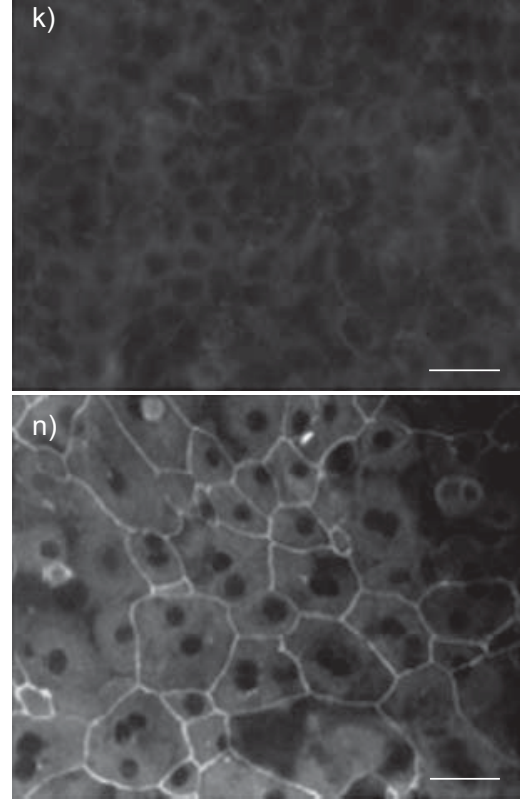

F-actin
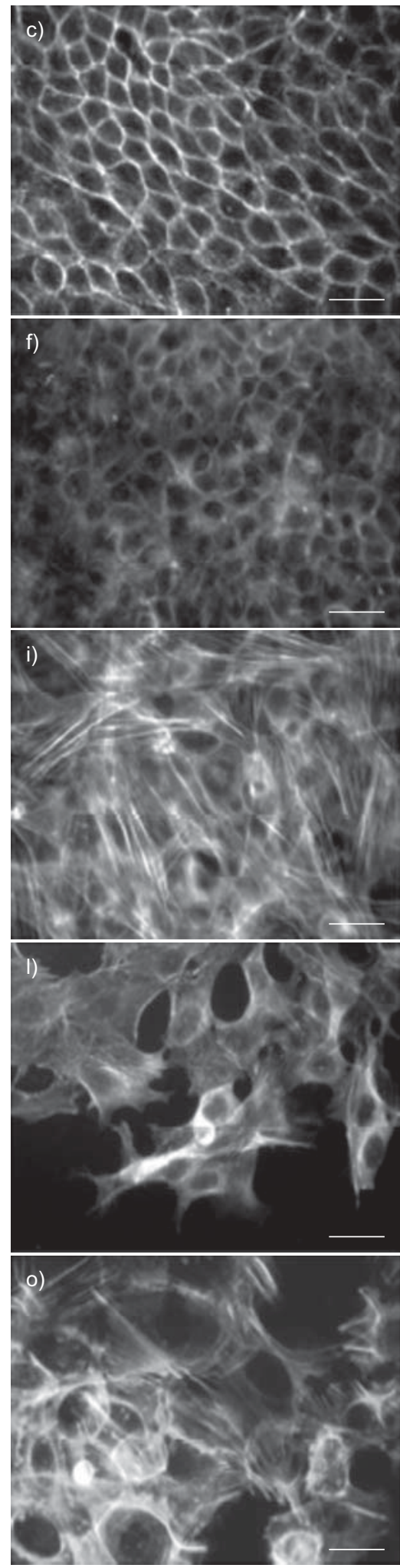

FIGURE 5. Localisation of cell adhesion molecules. a-c) 16HBE140- cells; $d-f$ ) NCl-H292 cells; $g-i)$ BEAS-2B cells; j-I) A549 cells; and m-0) primary bronchial epithelial cells (PBECs) derived from healthy donor I were seeded in duplicate in Lab-Teks and cultured to confluence (48-72 h). Zona occludens (ZO)-1 and E-cadherin were detected by immunofluorescence staining, and filamentous actin (F-actin) was visualised by rhodamine-labelled phalloidin staining. Representative results are shown. Scale bars $=25 \mu \mathrm{m}$. 


\begin{tabular}{|c|c|c|c|c|c|}
\hline & 16HBE140- & BEAS-2B & $\mathrm{NCl}-\mathrm{H} 292$ & A549 & PBECs \\
\hline Time to confluence ${ }^{\#} \mathrm{~h}$ & $30-50$ & $2.5-20$ & $30-40$ & $2.5-15$ & $2-10$ \\
\hline \multicolumn{6}{|l|}{$\mathrm{ZO}-1$ at $72 \mathrm{~h}$} \\
\hline Expression & High & Low & Moderate & Low & Moderate \\
\hline F-actin organisation & Cortical & Stress fibres & Cortical & Stress fibres & Stress fibres \\
\hline
\end{tabular}

PBEC: primary bronchial epithelial cell; ZO: zona occludens (tight junction protein); F-actin: filamentous actin. * : time taken to cover electrode following seeding at $7.5 \times 10^{4}$ cells $\cdot$ well $^{-1}$.
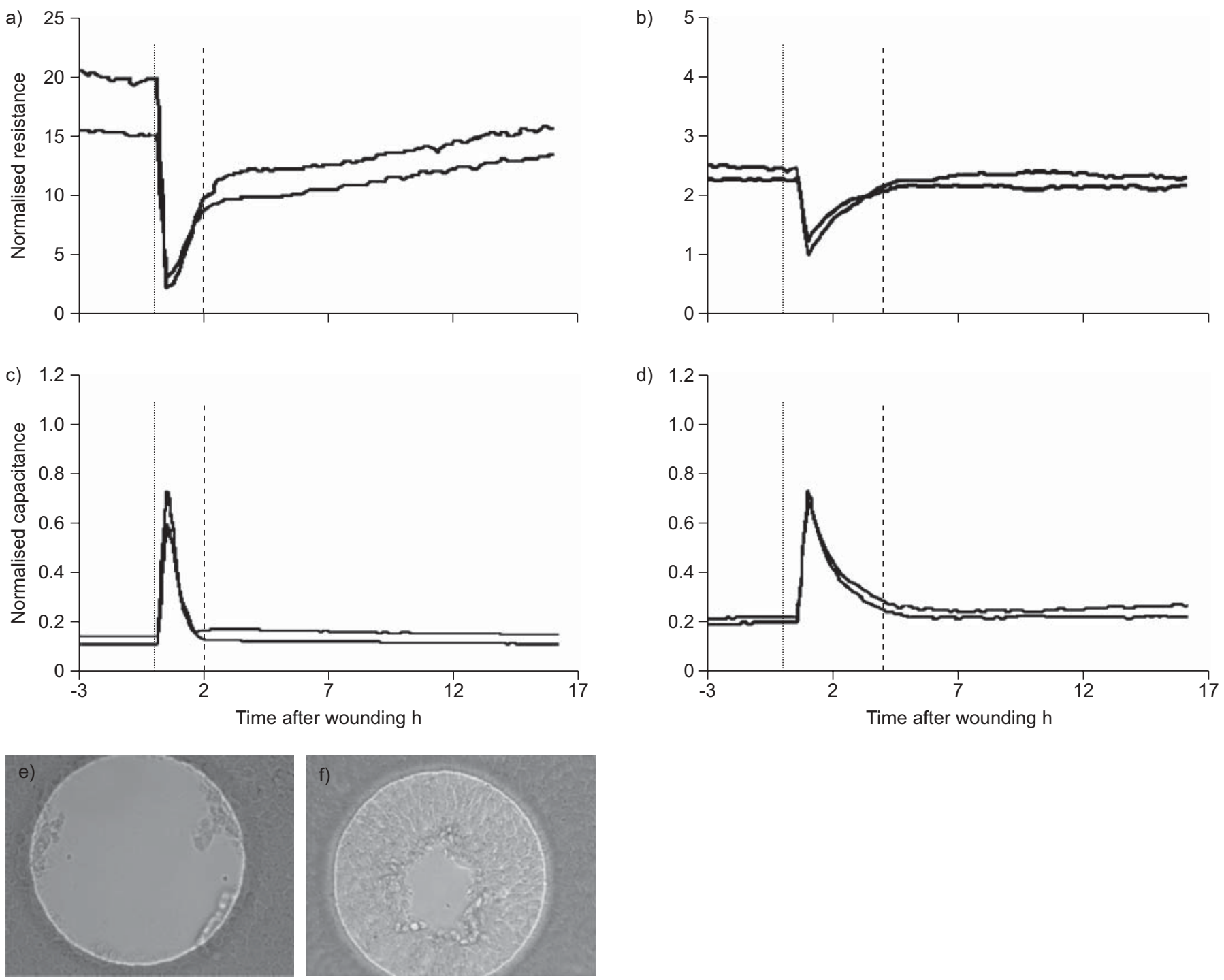

FIGURE 6. Differences between 16HBE140- and BEAS-2B cells in migration response and recovery of the epithelial barrier upon wounding by electroporation. Cells were seeded in duplicate into electric cell-substrate impedance sensing (ECIS) arrays. After $48 \mathrm{~h}$, cells were serum-deprived overnight. A wound was applied by electroporation. Resistance and capacitance at $400 \mathrm{~Hz}$ and $40 \mathrm{kHz}$, respectively, were measured using ECIS (both duplicates shown) over a time course of $20 \mathrm{~h}$, starting $3 \mathrm{~h}$ prior to wounding, in: a, c) 16HBE140- cells ( $\cdots \cdots: 0$ h; -----: 2 h); and b, d) BEAS-2B cells ( $\cdots \cdots: 0 \mathrm{~h}$; -----: 4 h). Immediately (e) and $1.5 \mathrm{~h}$ after wounding (f) of 16HBE140cells, the arrays were taken out, cells washed and photographs taken. 
wound repair. This should be kept in mind for translation to the in vivo situation. However, complete regeneration of the airway epithelium requires reconstitution of intercellular contacts. Here it is demonstrated that this is well reflected by ECIS measurements, since regeneration of the 16HBE140monolayer involved both migration and reconstitution of the barrier. In contrast, and as expected by their low-frequency resistance, BEAS-2B cells were inefficient at re-establishing cell-cell contacts upon wounding.

In summary, suggestive evidence is provided for the differential use of epithelial cell types in research on airway epithelial barrier function. The use of BEAS-2B and A549 cells may be of high translational value in studies on cell attachment mechanisms. However, the functional TJ deficits of the BEAS-2B, A549 and NCI-H292 cell lines preclude their use in studies on epithelial barrier formation. 16HBE14o- cells may serve as a suitable model for studying the behaviour of the bronchial epithelium with respect to barrier function, cell-cell contact formation, permeability, epithelial-to-mesenchymal transition and the reconstitution of intercellular junctions upon wounding.

\section{SUPPORT STATEMENT}

This study was supported by grant No. 3.2.05.039 from the Netherlands Asthma Foundation (Leusden, the Netherlands) and by GlaxoSmithKline.

\section{STATEMENT OF INTEREST}

A statement of interest for this study can be found at www.erj. ersjournals.com $/ \mathrm{misc} /$ statements.dtl

\section{REFERENCES}

1 Gumbiner B, Stevenson B, Grimaldi A. The role of the cell adhesion molecule uvomorulin in the formation and maintenance of the epithelial junctional complex. J Cell Biol 1988; 107: 1575-1587.

2 de Boer WI, Sharma HS, Baelemans SM, et al. Altered expression of epithelial junctional proteins in atopic asthma: possible role in inflammation. Can J Physiol Pharmacol 2008; 86: 105-112.

3 Holgate ST. The airway epithelium is central to the pathogenesis of asthma. Allergol Int 2008; 57: 1-10.

4 Trautmann A, Kruger K, Akdis M, et al. Apoptosis and loss of adhesion of bronchial epithelial cells in asthma. Int Arch Allergy Immunol 2005; 138: 142-150.

5 Lapperre TS, Sont JK, van Schadewijk A, et al. Smoking cessation and bronchial epithelial remodelling in COPD: a cross-sectional study. Respir Res 2007; 8: 85.
6 Kato Y, Hirano T, Yoshida K, et al. Frequent loss of E-cadherin and/or catenins in intrabronchial lesions during carcinogenesis of the bronchial epithelium. Lung Cancer 2005; 48: 323-330.

7 Winton HL, Wan H, Cannell MB, et al. Cell lines of pulmonary and non-pulmonary origin as tools to study the effects of house dust mite proteinases on the regulation of epithelial permeability. Clin Exp Allergy 1998; 28: 1273-1285.

8 Wegener J, Keese CR, Giaever I. Electric cell-substrate impedance sensing (ECIS) as a noninvasive means to monitor the kinetics of cell spreading to artificial surfaces. Exp Cell Res 2000; 259: 158-166.

9 Heijink IH, Kies PM, Kauffman HF, et al. Down-regulation of Ecadherin in human bronchial epithelial cells leads to epidermal growth factor receptor-dependent Th2 cell-promoting activity. $J$ Immunol 2007; 178: 7678-7685.

10 Borger P, Kauffman HF, Scholma J, et al. Human allogeneic CD2+ lymphocytes activate airway-derived epithelial cells to produce interleukin-6 and interleukin-8. Possible role for the epithelium in chronic allograft rejection. J Heart Lung Transplant 2002; 21: 567-575.

11 Heijink IH, Postma DS, Noordhoek JA, et al. House dust mitepromoted epithelial-to-mesenchymal transition in human bronchial epithelium. Am J Respir Cell Mol Biol 2010; 42: 69-79.

12 Lordan JL, Bucchieri F, Richter A, et al. Cooperative effects of Th2 cytokines and allergen on normal and asthmatic bronchial epithelial cells. J Immunol 2002; 169: 407-414.

13 Heijink IH, Vellenga E, Oostendorp J, et al. Exposure to TARC alters $\beta_{2}$-adrenergic receptor signaling in human peripheral blood $\mathrm{T}$ lymphocytes. Am J Physiol Lung Cell Mol Physiol 2005; 289: L53-L59.

14 Slebos DJ, Ryter SW, van der Toom M, et al. Mitochondrial localization and function of heme oxygenase- 1 in cigarette smokeinduced cell death. Am J Respir Cell Mol Biol 2007; 36: 409-417.

15 Woo PL, Cercek A, Desprez PY, et al. Involvement of the helixloop-helix protein Id-1 in the glucocorticoid regulation of tight junctions in mammary epithelial cells. J Biol Chem 2000; 275: 28649-28658.

16 Tunggal JA, Helfrich I, Schmitz A, et al. E-cadherin is essential for in vivo epidermal barrier function by regulating tight junctions. EMBO J 2005; 24: 1146-1156.

17 van Wetering S, Zuyderduyn S, Ninaber DK, et al. Epithelial differentiation is a determinant in the production of eotaxin- 2 and -3 by bronchial epithelial cells in response to IL-4 and IL-13. Mol Immunol 2007; 44: 803-811.

18 Fukuda $\mathrm{M}$, Nishida $\mathrm{T}$, Otori $\mathrm{T}$. Role of actin filaments and microtubules in the spreading of rabbit corneal epithelial cells on the fibronectin matrix. Cornea 1990; 9: 28-35.

19 Keese CR, Wegener J, Walker SR, et al. Electrical wound-healing assay for cells in vitro. Proc Natl Acad Sci USA 2004; 101: 1554-1559.

20 Soong HK, Dass B, Lee B. Effects of cytochalasin D on actin and vinculin in cultured corneal epithelial cells. J Ocul Pharmacol 1990; 6: 113-121. 